\title{
Adrenal response in very low birthweight babies after dexamethasone treatment for bronchopulmonary dysplasia
}

\author{
P C NG, M E BLACKBURN, K G BROWNLEE, J M H BUCKLER, * AND P R F DEAR \\ Department of Paediatrics and Child Health, University of Leeds, St James's University Hospital and *The \\ General Infirmary at Leeds
}

SUMMARY The tetracosactrin stimulation test was used to assess the adrenal responsiveness of 22 very low birthweight babies who had received a three week course of dexamethasone for the treatment of bronchopulmonary dysplasia. Five babies were studied in detail with blood samples taken for cortisol concentrations at 30 minute intervals for four hours. The tests were performed before, during, and after treatment with dexamethasone. A distinctive pattern of cortisol response to tetracosactrin was found among these babies, which was quite unlike that found in older children and adults. Using our pretreatment results as control data we conclude that there is undoubtedly evidence of modest suppression of the adrenal axis during dexamethosone treatment, although there is considerable recovery one month after stopping steroids. Basal cortisol concentrations, however, remained low in some cases, which may indicate the need for temporary corticosteroid replacement during severe illness.

Corticosteroids are being used increasingly for the treatment of bronchopulmonary dysplasia in preterm babies. If the British multicentre trial now underway reports favourably on the effectiveness of steroids in this condition, their use will undoubtedly increase further. We have already observed that a course of dexamethasone, in the dose of $0.6 \mathrm{mg} / \mathrm{kg} / \mathrm{day}$, can bring about a dramatic improvement in lung function and subsequent reduction in ventilatory requirements in many babies with bronchopulmonary dysplasia. Some cases, however, seem to relapse at the end of the one week course of dexamethasone usually prescribed. In an attempt to prevent relapse we have extended the course to three weeks, tapering the dose progressively after the first week. As the literature contains little, if any, information on the effect of steroids on the adrenal gland of very low birthweight babies, we have undertaken to perform a series of tetracosactrin stimulation tests on 22 babies (five studied in detail) whom we have treated with steroids during the past 18 months.

\section{Patients and methods}

The investigation was performed on 22 very low birthweight babies admitted to the regional neonatal unit at St James's University Hospital, Leeds. None of their mothers had received steroids during pregnancy. All the babies required ventilation from birth for respiratory distress syndrome and had developed bronchopulmonary dysplasia of sufficient severity to prevent them from coming off ventilatory support. Each baby was given a full three week course of dexamethasone: $0.6 \mathrm{mg} / \mathrm{kg} / \mathrm{day}$ for the first week, half of this dose in the second week, and a quarter of it in the third week.

Tetracosactrin stimulation tests were performed on day 0 , just before dexamethasone was started, at the end of week 1 and at the end of the three week course. If babies were still in the unit one month after steroid treatment finished (that is, week 7), a further test was performed. All tests were performed at $9 \mathrm{am}$. Adrenal stimulation was produced by intramuscular injection of $125 \mu \mathrm{g}$ of tetracosactrin (Synacthen, Ciba). In 17 babies, a standard test was performed, with blood samples taken immediately before, and 30 minutes after, tetracosactrin. Almost all samples were obtained from indwelling arterial lines but if this access was unavailable when a sample was needed, capillary blood was used.

In five babies, who had indwelling arterial lines on 
each occasion a test was due, we performed a prolonged test, with samples taken at 30 minute intervals for four hours.

Fully informed parental consent was obtained in each case. The study was approved by the hospital clinical research (ethics) committee.

Plasma was extracted and stored at $4^{\circ} \mathrm{C}$. All samples were analysed within 48 hours. Plasma

Table 1 Clinical data and plasma cortisol concentration at 0 and 30 minutes in babies studied with conventional tetracosactrin test $(n=17)$

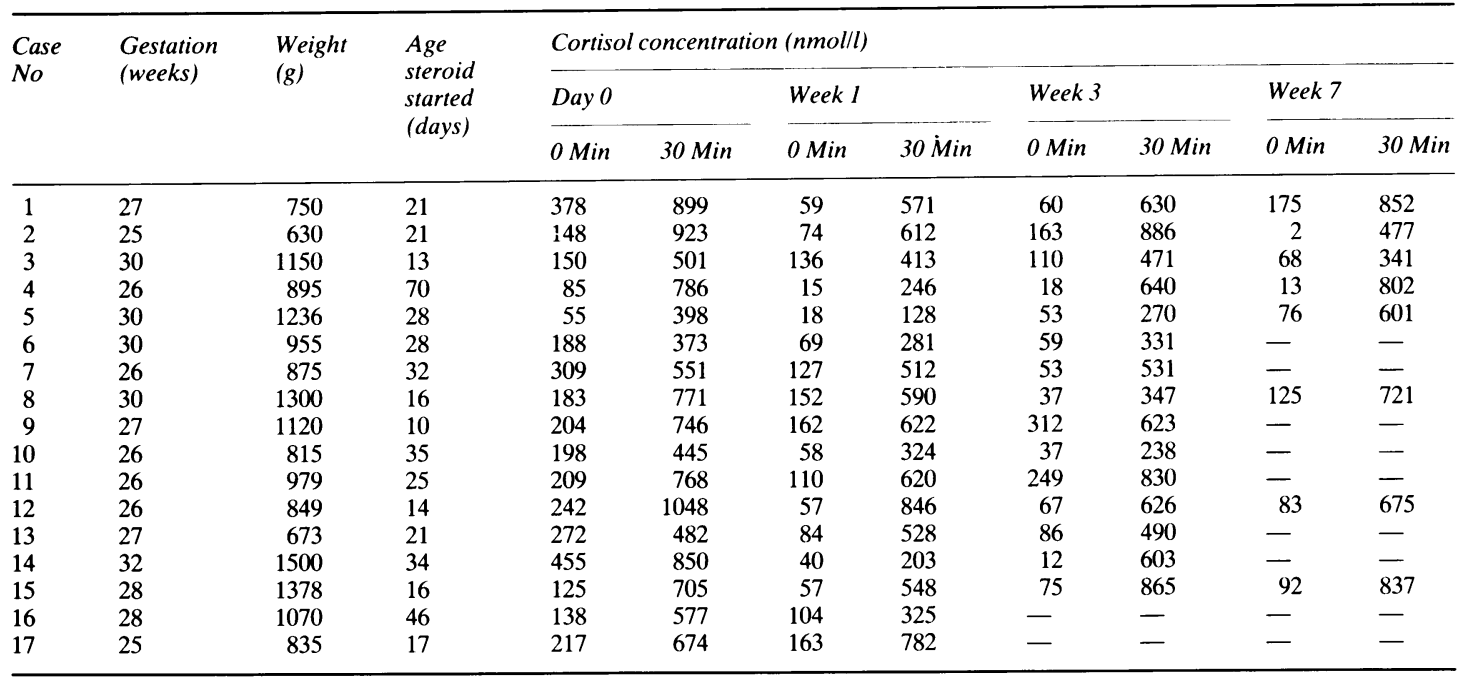

Table 2 Clinical data and cortisol concentrations in the five babies studied with an extended tetracosactrin test

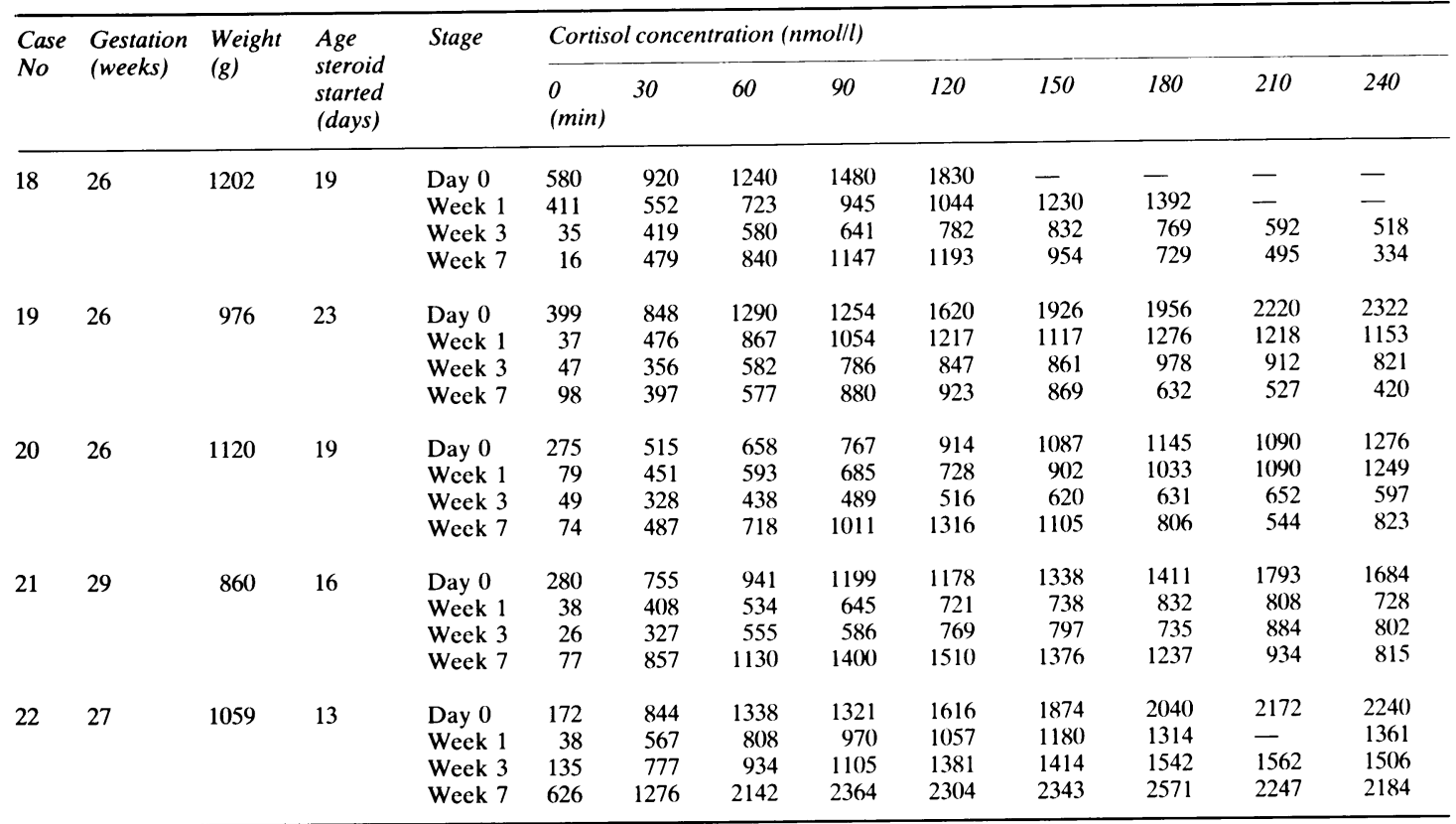


cortisol was measured in duplicate using a fluorescence polarisation immunoassay technique with the Abbott diagnostic TDx analyser. Cross reactivity with endogenous steroids was: corticosterone $4.5 \%$ and cortisone $1 \cdot 1 \%$, and with synthetic steroids: prednisolone $23.8 \%$, prednisone $0.4 \%$, and dexamethasone $0.6 \%$.

Paired $t$ tests and simple regression were used for statistical analysis where appropriate.

\section{Results}

The clinical data and the results of the tetracosactrin test at various stages are summarised in tables 1 and 2 . Table 1 concerns the 17 babies who had a standard test and table 2 those who had the extended test. Data is missing as follows: one patient in the extended study group did not have his cortisol concentration monitored for the full four hours as we were not initially aware of the prolonged nature of the response; two patients returned to their referring hospitals before the course of dexamethasone was completed and so missed both the week 3 and the final tetracosactrin test, and six others went home before the last test was due; two patients died, one before the final test was performed.

Fig 1 shows the mean plasma cortisol responses of the five babies studied in detail, at the four different stages in relation to dexamethasone treatment. On day 0 , the cortisol concentration rose steeply and was still rising at four hours. The cortisol responses on weeks 1 and 3 were diminished in magnitude in proportion to the duration of dexamethasone treatment and the curves showed signs of becoming asymptotic after about 120 minutes. One month after stopping steroid treatment the cortisol responses consistently peaked at 120 minutes and then declined rapidly.

These different patterns of response make it difficult to fix on an ideal time after tetracosactrin stimulation at which to measure the cortisol concentration. Because we felt that the concentration at 120 minutes was probably the most informative, we were interested to see how the cortisol at this time correlated with the conventional 30 minute and 60 minute concentrations. Simple regression analysis was used to examine the correlation. Both the 30 minute $(n=20)(f i g)$ and 60 minute $(n=20)$ cortisol concentrations, at the four stages of treatment, correlated well with the corresponding 120 minute concentration $(n=20)$; the correlation coefficients $(r$ values) were 0.94 and 0.95 respectively $(p<0.00001)$. The correlation between 30 and 60 minutes was also excellent $(r=0 \cdot 96, p<0 \cdot 00001)$.

When the cortisol concentrations before dexamethasone treatment (day 0) were compared with later concentrations in the same baby, we found that after one and three weeks of dexamethasone the cortisol concentration at all sampling times, up to 180 minutes, was significantly lower than before dexamethasone treatment was started $(\mathrm{p}<0.035$, paired $t$ test). In general, the results in the week 3 test showed a greater suppression than in week 1 . By contrast, all week 7 results were not significantly different from control $(p>0.08$, paired $t$ test).

Paired $t$ tests were also used to compare the increment in cortisol concentration (that is, the increase above prestimulation concentration) between the test before dexamethasone and later tests. Significance differences were found on very few occasions-at $90,120,150,180,210$, and 240 minutes in week $3(\mathrm{p}<0.03)$; at 30 minutes in week 1 $(p<0.05)$, and at 90 minutes in week $7(p<0.05)$.

When the basal cortisol values of all babies were pooled and compared at various times in relation to

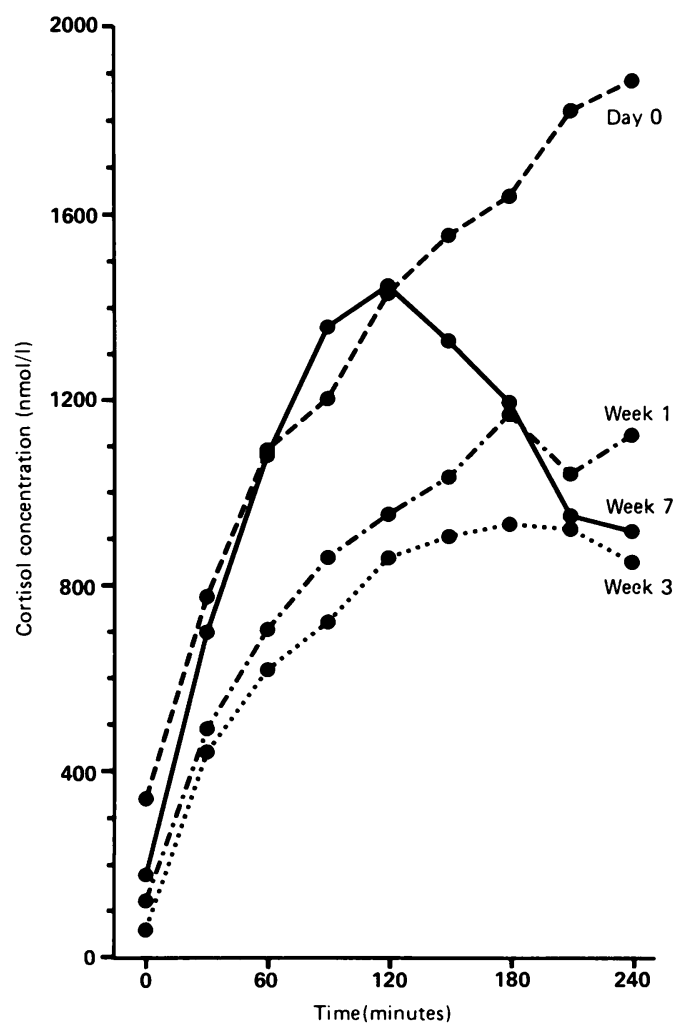

Fig 1 Mean plasma cortisol concentrations of five babies studied by the extended tetracosactrin test at four stages in relation to dexamethasone treatment. 


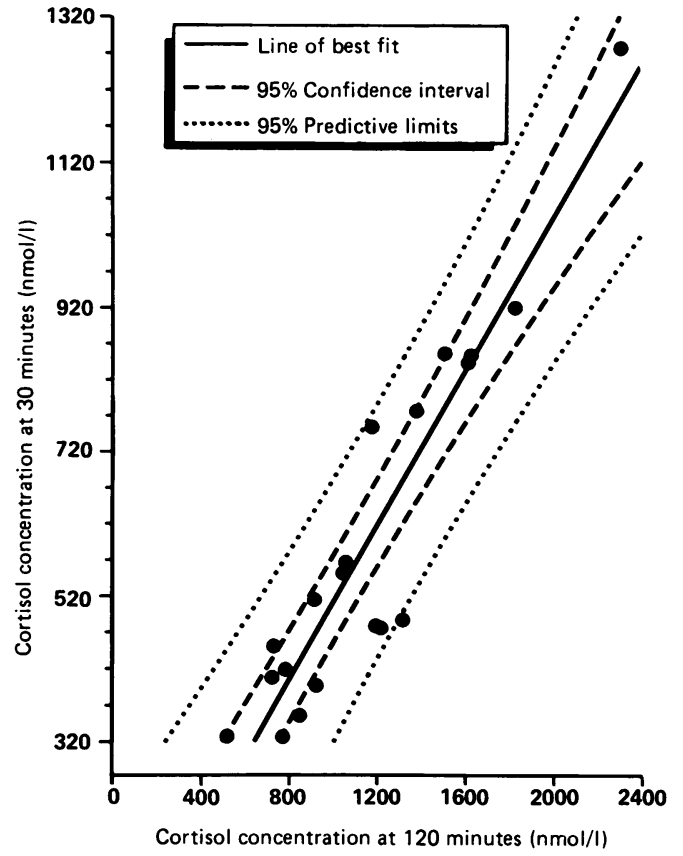

Fig 2 Correlation between cortisol concentrations at 30 and 120 minutes.

dexamethasone treatment, they were seen to fall from a mean of $239 \mathrm{nmol} / \mathrm{l}$ before treatment to 95 and $84 \mathrm{nmol} / \mathrm{l}$, after one and three weeks of dexamethasone respectively $(p<0 \cdot 001)$. There was a difference between the mean pretreatment basal concentration of $239 \mathrm{nmol} / \mathrm{l}$ and the mean basal concentration of $117 \mathrm{nmol} / \mathrm{l}$ one month after the end of treatment, but this was not significant $(p=0.08)$.

The correlation between the dose of tetracosactrin, expressed per $\mathrm{kg}$ body weight, and both the 30 minute and incremental cortisol response before dexamethasone treatment was examined. Correlation coefficients of 0.06 and 0.11 respectively were obtained $(p=0.78$ and 0.63$)$.

Correlations were also performed between 30 minute cortisol concentration and both gestational age and postconceptional age. The coefficients were -0.29 and -0.32 respectively $(p=0.20$ and $0 \cdot 16)$. The correlation coefficients between the incremental response, and gestational and postconceptional age were -0.26 and -0.25 respectively $(p=0.27$ and $0 \cdot 29$ ).

\section{Discussion}

Suppression of adrenal gland responsiveness is one of the most serious side effects of steroid treatment, and a case of adrenal insufficiency has been reported in a neonate whose mother had received long term steroid treatment during pregnancy. ${ }^{\circ}$ As dexamethasone is now being prescribed for the treatment of bronchopulmonary dysplasia, an understanding of its effect on adrenal responsiveness in very low birthweight babies is important. A literature search shows that information on adrenal function in this group of patients is scanty and at times confusing. The main source of confusion is the variable methodology used for performing tetracosactrin stimulation tests, both in terms of the size of the tetracosactrin dose and the timing of the blood sample taken after stimulation..$^{2-9}$ As a result there is in our opinion no reliable reference range against which to assess the adequacy of the adrenal response of very low birthweight babies. We chose to standardise on $125 \mu \mathrm{g}$ of tetracosactrin, which is a large weight related dose for our smallest babies, in order to produce maximum stimulation of the adrenal gland. This was to avoid overdiagnosing adrenal suppression, which we felt was a risk in view of the paucity of knowledge about the adrenal response in such immature babies as were included in our study. In fact, our results showed no significant correlation between the dose of tetracosactrin $/ \mathrm{kg}$ body weight and either the 30 minute cortisol concentration or increment.

We have sought to overcome some of the difficulties of interpretation of the test by studying a small number of babies in detail, so as to delineate the cortisol response more thoroughly. Also by performing tetracosactrin tests immediately before starting dexamethasone, we aimed to provide a reference point against which later responses might be judged.

In the extended study group we observed a progressive change in the shape of the response curves (fig 1), and several explanations can be offered. One possibility is that there was no effect of dexamethasone and we simply observed the normal maturational changes of the adrenal gland during late fetal and early neonatal life. To examine this in more detail we looked for a correlation between adrenal response and both gestational and postconceptional age before dexamethasone treatment. The fact that there was no significant correlation between either gestational or postconceptional age and any aspect of cortisol response suggests that maturational changes during this period are not the explanation for the observed changes in response. Another explanation, which is more plausible and could be contributory, is that the change in response might be stress related, as many of the babies were becoming progressively less ill as time went by. In this respect 
it is interesting to note that the sickest baby in the study (table 2, case 22), who did not respond to dexamethasone and who died of bronchopulmonary dysplasia, had the greatest cortisol response to tetracosactrin at weeks 3 and 7 . The most important factor in our view, however, is the effect of dexamethasone on the hypothalamic-pituitary-adrenal axis, with partial recovery after a month without steroids.

Although in most reports either the 30 or 60 minute concentrations are considered suitable end points for the tetracosactrin test, ${ }^{2-5} 7-9$ we suspect from our data that they do not represent the most appropriate times to use in this age group. Results from our extended study indicate that concentrations at these times do not represent peak concentrations. We suggest that the 120 minute concentration might represent the best compromise in this group of patients. This is because at week 7 the cortisol concentration consistently peaked at this time, and at the earlier stages (that is, weeks 1 and 3 ) it was very close to its asymptote. At week 0 , although far from the peak, the 120 minute concentration at least allows time for a substantial rise in plasma cortisol to occur. It was reassuring to us, and should be to other investigators, to find that the often used 30 and 60 minute concentrations correlate very well with that found at 120 minutes (fig 2).

The fall in basal cortisol concentration after one and three weeks of dexamethasone was most likely due to suppression of the hypothalamic-pituitary higher centres by dexamethasone. The mean basal concentration four weeks after stopping dexamethasone was also lower than it was before starting dexamethasone, although not significantly so. As there was no lessening of the response after tetracosactrin stimulation at week 7 , this observation raises the possibility of a more severe and prolonged effect of steroid treatment on higher centres.

Tetracosactrin stimulation tests are conventionally evaluated by comparison with reference ranges for representative populations. Commonly advocated criteria for a satisfactory response in children are an increment of $>200 \mathrm{nmol} / \mathrm{l}$ or a 30 minute value of $>500 \mathrm{nmol} / \mathrm{l} .{ }^{10} \mathrm{By}$ the first of these criteria one of our 22 patients would be judged underresponsive before dexamethasone, three of them after one week's treatment, and none after three and seven weeks. By the standard of a 30 minutes concentration $>500 \mathrm{nmol} / \mathrm{l}$, four out of 22 would be judged under-responsive before dexamethasone, 10 out of 22 after one week's treatment, 10 out of 20 after three weeks' treatment, and five out of 13 at week 7. As it is unlikely, from our extended data, that any of our babies had reached their peak cortisol concentrations at the time of the 30 minute sampling, our data probably overestimate the incidence of under-responsiveness as judged by the criterion of the 30 minute peak concentration. In fact the data from the extended study group show that none of these five cases had a response which failed to fulfil either of the above criteria at any stage.

When comparisons of incremental response were made using the pretreatment concentration (day 0) as control, the response was significantly reduced only at week 3 . By contrast, the absolute cortisol concentrations in week 1 and 3 were significantly lower than on day 0 at all times after stimulation. At week 7 , neither the increments nor the absolute concentrations were significantly different from those before treatment. Interpretation of results is undoubtedly complicated by the lack of universal agreement on which of the three pieces of information obtained from the tetracosactrin test-the basal cortisol, the peak cortisol, or the increment-is the best measure of adrenocortical responsiveness. ${ }^{11}$ One of the problems is the extent to which the three measures are interdependent. It seems clear, however, that in our babies some degree of adrenal suppression had taken place after one week of treatment and appeared to be most severe at the end of the three week course. One month after stopping steroids the adrenal gland was probably responding within normal limits. Throughhout the treatment and recovery period none of the babies showed any signs of clinical or biochemical adrenal insufficiency.

We conclude that a course of dexamethasone as described in this study is relatively safe in terms of adrenal suppression and, at first glance, it would seem unnecessary to give steroid replacement at times of stress. The low basal cortisol concentrations found in some patients four weeks after stopping steroids, however, suggest that there may still be a degree of suppression of the hypothalamic and pituitary higher centres, which could indicate the need for steroid or adrenocorticotrophic hormone treatment during severe illness.

\footnotetext{
References

1 Grajwer LA, Lilien LA, Pildes RA. Neonatal subclinical adrenal insufficiency. Result of maternal steroid therapy. JAMA 1977;238:1279-80.

2 Noguchi A, Reynolds JW. Serum cortisol and dehydroepiandrosterone sulfate responses to adrenocorticotropin stimulation in premature infants. Pediatr Res 1978;12:1057-61.

3 Barnhart BJ, Carlson CV, Reynolds JW. Adrenal cortical function in the postmature fetus and newborn infant. Pediatr Res 1980;14:1367-9.

4 Arad I, Landau H. Adrenocortical reserve of neonates born of long-term, steroid-treated mothers. Eur J Pediatr 1984;142:27980 .

5 Thomas S, Murphy JF, Dyas J, Ryalls M, Hughes IA. Response to ACTH in the newborn. Arch Dis Child 1986;61:57-60.

${ }^{6}$ Gutai J, George R, Koeff S, Bacon GE. Adrenal response to
} 
physical stress and the effect of adrenocorticotropic hormone in newborn infants. $J$ Pediatr 1972;81:719-25.

7 Nwosu UC, Johnson L, Bongiovanni AM, Boggs TR, Wallach EE. Adrenocortical response to ACTH stimulation in postmature newborns. Obstet Gynecol 1978;52:213-4.

8 Ohrlander S, Gennser G, Nilsson KO, Eneroth P. ACTH test in neonates after administration of corticosteroids during gestation. Obstet Gynecol 1977;49:691-4.

9 Arnold JD, Leslie GI, Williams G, Rack P, Silink M. Adrenocortical responsiveness in neonates weaned from the ventilator with dexamethasone. Aust Paediatr J 1987;23:227-9.

10 Belton NR. Biochemical and physiological tables and function tests. In: Forfar JO, Arneil GC, eds. Textbook of paediatrics. Vol 2, 3rd ed. Edinburgh: Churchill Livingstone, 1984: 1977-2007.

" May ME, Carey RM. Rapid adrenocorticotropic hormone test n practice. Am J Med 1985;79:679-84.

Correspondence to Dr PRF Dear, Department of Paediatrics and Child Health, St James's University Hospital, Beckett St, Leeds LS9 7TF.

Accepted 3 July 1989 